\title{
Evaluation of the Activity of Neutrophils and Monocytes in Diabetic Patients With Sepsis, Can Surface Antigens HLA-DR and CD64 Be Useful as Prognostic Factors?
}

\author{
Kalliopi Lekka ${ }^{\mathrm{a}}$, Markos Marangos ${ }^{\mathrm{b}}$, Nikolaos Roupas ${ }^{\mathrm{c}}$, Marina Karakantza ${ }^{\mathrm{d}}$, \\ Charalampos Gogos ${ }^{\mathrm{a}}$, Dimitrios Velissaris ${ }^{\mathrm{a}}, \mathrm{e}$
}

\begin{abstract}
Background: Patients with diabetes mellitus (DM) exhibit alterations in their immune response when infected by several types of micro-organisms. The increased susceptibility of diabetics to infections is particularly related to abnormalities in the function of neutrophils such as chemotaxis, adhesion and intracellular killing, leading to increased mortality rates. Aims of the study were to assess the phagocytic activity and the expression of antigens HLA-DR and CD64 of monocytes and neutrophils in diabetics with sepsis and evaluate their significance as prognostic factors.
\end{abstract}

Methods: This is an observational prospective study conducted in a tertiary medical center, referring to a population of 51 diabetic patients who were treated for sepsis. Samples of whole blood were received from the selected patients and were evaluated for the expression of surface antigens HLA-DR and CD64 on monocytes and neutrophils, and for their phagocytic activity as well.

Results: Alterations in the phagocytic activity were found in the diabetic patients who developed sepsis, and these were addressed as an elevation in the expression of CD64 on monocytes (CD64M), and a reduction in the expression of HLA-DR on monocytes (HLA-DRM) at least in the initial phase of the acute infection. A significant elevation was also noticed in the phagocytosis rate of both neutrophils and monocytes on day of admission. Survivors had higher rates of both CD64 and HLA-DR on monocytes when compared to non-survivors. No correlation was found between glycemic control, values of inflammatory markers on admission, phagocytosis rate and the survival of diabetics with sepsis. A reduced expression of CD64O, HLA-DRM and the co-expression of CD64/HLA-DR on monocytes in the initial

Manuscript submitted December 11, 2019, accepted February 1, 2020

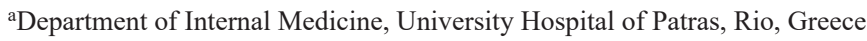
bepartment of Infectious Diseases, University Hospital of Patras, Rio, Greece 'Department of Endocrinology, University Hospital of Patras, Rio, Greece dDivision of Hematology, Department of Internal Medicine, University Hospital of Patras, Rio, Greece

${ }^{\mathrm{e} C}$ Corresponding Author: Dimitrios Velissaris, Department of Internal Medicine, University Hospital of Patras, Rio 26504, Greece. Email: dvelissaris@ upatras.gr

doi: https://doi.org/10.14740/jocmr4068 phase of sepsis and poor glycemic control (hemoglobin A1c (HbAlc) $>8.5$ ) was found.

Conclusions: In the present study of diabetic patients with sepsis the phagocytic activity of neutrophils and monocytes is elevated at the initial phase of an acute infection and only the values of CD64 and HLA-DR on monocytes were significantly related to outcome. Further evaluation of these results with large prospective studies is warranted.

Keywords: Sepsis; Diabetes; Phagocytosis; HLA-DR; CD64; Monocytes; Neutrophils; Mortality

\section{Introduction}

Sepsis, a syndrome of clinical and biochemical abnormalities induced by infection, remains a major public health issue, still with high mortality rates. The incidence of sepsis is increasing worldwide, as several populations with underlying comorbidities are affected $[1,2]$. In particular, patients with diabetes are suffering from the highest infection rates.

Several mechanisms are associated with the increased susceptibility of the diabetic patients when infected. Excessive innate and adaptive immune system changes that the human body cannot overcome are taking place as part of the combined underlying chronic immune suppression of diabetes and the sepsis-induced immune alterations [3].

The role of neutrophils is crucial for the containment and the eradication of the micro-organisms. These cells are part of the innate immune system, producing pro- and anti-inflammatory cytokines and growth factors, and also are involved in the regulation of the inflammatory response [4]. Diabetes is characterized by some specific deficits in the cell migration to the inflammatory sites, alterations in phagocytosis and release of lytic proteases, production of reactive oxygen species (ROS), altered apoptosis and an excessive release of tumor necrosis factor (TNF), interleukin (IL)-1 $\beta$, and IL-8 after stimulation by lipopolysaccharide (LPS). The last is related to cell death and increased susceptibility to micro-organisms [5, 6]. Aims of the study are to investigate the phagocytic activity and the expression of antigens HLA-DR and CD64 on monocytes and neu- 
trophils in diabetic patients suffering from sepsis and evaluate their prognostic validity.

\section{Materials and Methods}

The study was conducted in the Internal Medicine Department of the University Hospital of Patras, Greece, during the period from December 2016 to December 2017. All enrolled patients gave consent for participating in the study and the University Hospital Ethics Committee approved the protocol. During the study period, 51 diabetics (one with type 1 diabetes and 50 with type 2 diabetes) with sepsis, 22 women and 29 men, were enrolled. Fourteen of them had excellent glycemic control $(\mathrm{HbA} 1 \mathrm{c} \leq 7$ or $\mathrm{HbA} 1 \mathrm{c} \leq 53 \mathrm{mmol} / \mathrm{mol})$, while the rest had moderate $(7 \leq \mathrm{HbAlc} \leq 8.5$ or $53<\mathrm{HbAlc} \leq$ $69.40 \mathrm{mmol} / \mathrm{mol}$ ) or bad glycemic control (HbA1c $\geq 8.5$ or $\mathrm{HbA} 1 \mathrm{c}>69.40 \mathrm{mmol} / \mathrm{mol}$ ). With regards to treatment of diabetes, 16 patients were on insulin, 25 were on oral medication (metformin, sulfonylurea, pioglitazone, dipeptidyl peptidase-4 (DPP-4) inhibitors), two were on a combined treatment and eight did not follow any treatment. Three of our patients came along with diabetic ketoacidosis as a result of their underlying infection. Eleven patients had been diagnosed with diabetes mellitus (DM) in the past 5 years, whereas 24 had diabetes for 10 years or more. As far as diabetic complications were concerned, four patients suffered from peripheral angiopathy and five from diabetic nephropathy. Regarding the existence of other chronic diseases, 28 patients with diabetes had arterial hypertension, hyperlipidemia was present in 12, 12 patients had ischemic heart disease, six had congestive heart failure, eight had atrial fibrillation, nine had chronic obstructive pulmonary disease (COPD), four were obese, and eight had a history of stroke. Exclusion criteria for participating in the study were known renal, hepatic or any autoimmune disease, treatment with steroids or any immuno-effective agent, diabetics with known white blood cells abnormalities, and those without complete data during the study period.

The definition of sepsis was based on the documented positive culture infection or presumed infection accompanied by two or more of the criteria of systemic inflammatory response syndrome (SIRS). The diagnostic workup consisted of clinical assessment in combination to evaluation of inflammatory markers (white blood count, C-reactive protein), biochemical panel, blood, urine or any other suspicious site specimen cultures, and specific diagnostic procedures when necessary to identify the cause of sepsis (e.g. computed tomography, magnetic resonance imaging).

\section{HLA-DR, CD64 and phagocytosis measurements}

In order to measure the phagocytic activity of neutrophils and monocytes, $5 \mathrm{~mL}$ of peripheral blood was obtained in heparinized tubes. The determination of HLA-DR and CD64 expression on neutrophils and monocytes was operated through dual-colour cytometry flow analysis. More specific, in $100 \mu \mathrm{L}$ of whole blood we added $20 \mu \mathrm{L}$ of HLA-DR fluorescein iso- thiocyanate (FITC) monoclonal antibody and $20 \mu \mathrm{L}$ of CD64 phycoerythrin (PE) antibody. In another sample of $100 \mu \mathrm{L}$ of whole blood of the same patient we added $20 \mu \mathrm{L}$ of isotypic monoclonal antibody marked with FITC and $20 \mu \mathrm{L}$ of isotypic monoclonal antibody marked with $\mathrm{PE}$, in order to mark our controls. We used as control group 30 diabetic volunteers matched by age and gender. After 30-min incubation period in room temperature, erythrocytes were lysed and leukocytes were fixed with the Multi-Q-Prep system (Coulter, Nyon, Switzerland). At least 10,000 cells from each sample were analyzed on the Epics XL (Coulter) flow cytometer and data were processed with XL2 software. Analysis was performed in a scattergram gate of cells with intermediate side and forward scatter. Results were expressed as percentages of CD64 and HLA-DR positive neutrophils and monocytes, and as arbitrary units (mean fluorescence intensity (MFI)) as well.

The phagocytic activity of neutrophils and monocytes was also measured on the Epics XL (Coulter) flow cytometer, after incubation of $100 \mu \mathrm{L}$ of whole blood sample with $20 \mu \mathrm{L}$ of Escherichia coli (E. coli)-FITC, quenching, lysing and fixation, washing and DNA staining using the Glucotope Phagotest. Results were expressed as percentages of neutrophils and monocytes that appeared on the area marked with the green fluorochrome (FITC).

\section{Statistical analysis}

All statistical procedures were performed using SPSS 24.0 for Windows (IBM SPSS Statistics, IBM software, IBM Corp., Armonk, NY, USA). Test for normality was done using Kolmogorov-Smirnov test. All variables are expressed as mean \pm standard deviation (SD), regardless of their distribution.

Comparison of mean values between subgroups was performed using the independent $t$-test or one-way analysis of variance (ANOVA) when data distribution was normal and Mann-Whitney or Kruskall-Wallis test when the continuous variables were non-normally distributed. When appropriate, the comparisons were adjusted to the effect of additional variables (age, body mass index (BMI), body fat) via the general linear model. Variables that did not follow normal distribution were log-transformed to apply the general linear model. The comparison between diabetics with or without sepsis was controlled for the effect of glycemic control applying two-way ANOVA. Two-tailed statistical significance was set at 5\%.

Logistic regression analysis was implemented to assess the association of specific variables with the patient outcome. Correlations with a critical value of $\mathrm{P}<0.05$ were considered significant.

\section{Results}

Of the 51 diabetic patients with sepsis, 40 (78.43\%) survived and $11(21.56 \%)$ died during hospitalization. Five of the nonsurvivors died within the first 7 days of hospitalization. No special findings in the studied parameters in comparison to those that died later were observed. Three patients were trans- 
Table 1. Type of Infection

\begin{tabular}{ll}
\hline Infection & Number of patients \\
\hline Respiratory & $14(27.45 \%)$ \\
Urinary tract & $14(27.45 \%)$ \\
Osteomyelitis & $3(5.88 \%)$ \\
Soft tissues & $12(23.52 \%)$ \\
Abdomen & $8(15.68 \%)$ \\
\hline
\end{tabular}

ferred to the intensive care unit, none of whom survived. The etiology of sepsis was respiratory infection $(n=14)$, urinary tract infection $(\mathrm{n}=14)$, infection of the hepato-biliary tract $(\mathrm{n}$ $=8)$, infection of the skin and soft tissues $(\mathrm{n}=12)$, and osteomyelitis $(\mathrm{n}=3)$ (Table 1$)$.

Only in 19 cases the related causative micro-organism was isolated. The cultures were positive for $E$. coli in eight cases $(\mathrm{n}=8)$, Klebsiella pneumoniae $(\mathrm{n}=2)$, Staphylococcus aureus $(\mathrm{n}=5)$, Staphylococcus epidermidis $(\mathrm{n}=2)$, Citrobacter $(\mathrm{n}$ =1) and one patient had poly-microbial infection with Pseudomonas aeruginosa, Klebsiella pneumoniae and Proteus mirabilis, all isolated from rectum abscess.

Statistical analysis showed that diabetic patients with sepsis had higher rates of CD64 expression on monocytes on day 0 and phagocytosis in both monocytes and neutrophils on admission day (Table 2).

When comparing the studied parameters between survivors and non-survivors, the only statistically significant characteristic was the CD64 and HLA-DR expression on monocytes on day 7 after admission, which was elevated in the survivor group $(\mathrm{P}=0.023$ and $\mathrm{P}=0.026)$.

All results referring to survivors and non-survivors of the group of diabetics with sepsis are shown in Tables 2, 3 and 4.

\section{Discussion}

Sepsis is now defined as a life-threatening organ dysfunction caused by a dysregulated response of the human to an infection [7]. A number of abnormal immunological responses are generated during the progression of the sepsis syndrome, affecting the production and function of the innate and adaptive immune cells, leading to homeostatic disorders. The sepsis-associated immune defects are associated with increased mortality rates.

$\mathrm{DM}$ is one of the most frequent metabolic distortions predisposing for infectious diseases. Rare bacteria, atypical courses and frequent complications of infections are resulting in increased mortality. A number of underlying mechanisms such as the low-grade inflammatory status in DM type 2 (T2DM), the prolonged exposure to glucolipotoxicity and the increased oxidative stress are predisposing to the increased susceptibility to infections. Corner stone is the influence of glycemia on polymorphonuclear cell functions. Contributing factors are altered cellular and humoral immune deficiencies with alterations in neutrophil function and $\mathrm{T}$ cell response, poor blood supply to tissues, nerve damage and metabolic alterations [8-13].

The autoimmune perspectives in T2DM are associated with the presence of auto-antibodies, also including the selfreactive $\mathrm{T}$ cells or defects in regulatory $\mathrm{T}$ cells (Tregs). One contributor to the autoimmune activation in T2DM seems to be the chronic inflammatory state. Due to this persistent inflammation, tissue destruction leads to the triggering of an autoimmune response of cryptic self antigens, which in turn accelerates $\beta$-cell death. An increased secretion of inflammatory cytokines from both innate and adaptive immune system components, specifically macrophages and self-reactive $\mathrm{T}$ cells, occurs. However, the extent to which inflammation overlaps with autoimmunity is not known [14].

Neutrophils are the most integral cells of innate function, but in diabetes, they show defects in almost all functions such as migration to inflammatory sites, phagocytosis, release of lytic proteases, production of ROS and apoptosis. They can also eliminate a wide range of microbes by forming neutrophil extracellular traps (NETs). In sepsis, among other dysfunctions there are recognized elevated NET concentrations and these are related to severity of the syndrome and organ dysfunction. The increased tendency of neutrophils to form NETs in both diabetes and sepsis is recognized but how this contributes to worsen outcomes is unknown [10, 15-18].

The main implication on monocytes and to a lesser extent macrophages is reduced antigen presentation related to diminished HLA-DR cell surface expression [19]. In sepsis, an overall immune suppressive monocyte phenotype is well recognized [20]. When a diabetic is exposed to an acute infection, it is not clear enough how monocyte and macrophage populations are changing and how these changes are affected by a baseline obesity and chronic inflammation.

Although DM can massively deteriorate the course of an infection, we should not forget that sepsis as well can be devastating for the control of blood glucose levels. There is a rather bidirectional relationship between those two entities and it would be unfair to solely incriminate diabetes for bad outcomes in a septic patient.

In the present study we sought to evaluate the phagocytic activity of neutrophils and monocytes of diabetic patients suffering from sepsis. We used as markers of this activity the expression of CD64 on monocytes/neutrophils (CD64M/ CD64O) and the expression of HLA-DR on monocytes/neutrophils (HLA-DRM/HLA-DRO), in combination with the phagocytic capacity of both populations when exposed to a specific pathogen (E. coli).

Flow cytometry showed a decreased expression of CD64M on day 7 since admission, whereas the expression of CD64O was reduced on day 3 but elevated on day 7 of hospitalization. Regarding the expression of HLA-DRM and HLA-DRO, we found a reduction on day 3 of hospitalization, followed by a return to the admission levels regarding monocytes.

The current knowledge regarding pathophysiology of sepsis encompasses a two-stage syndrome with a pro-inflammatory phase lasting from the first 3 to 5 days followed by an anti-inflammatory phase. In our study the expression of both CD64M and CD64O was reduced during the initial phase of sepsis. Similarly, the expression of HLA-DRM and the expression of HLA-DRO are at the lower levels at the early stages.

We also found that the diabetics with sepsis who survived were those with an increased expression of the cell surface 
Table 2. Analysis of Predictors of Mortality Between Survivor and Non-Survivor Diabetics With Sepsis

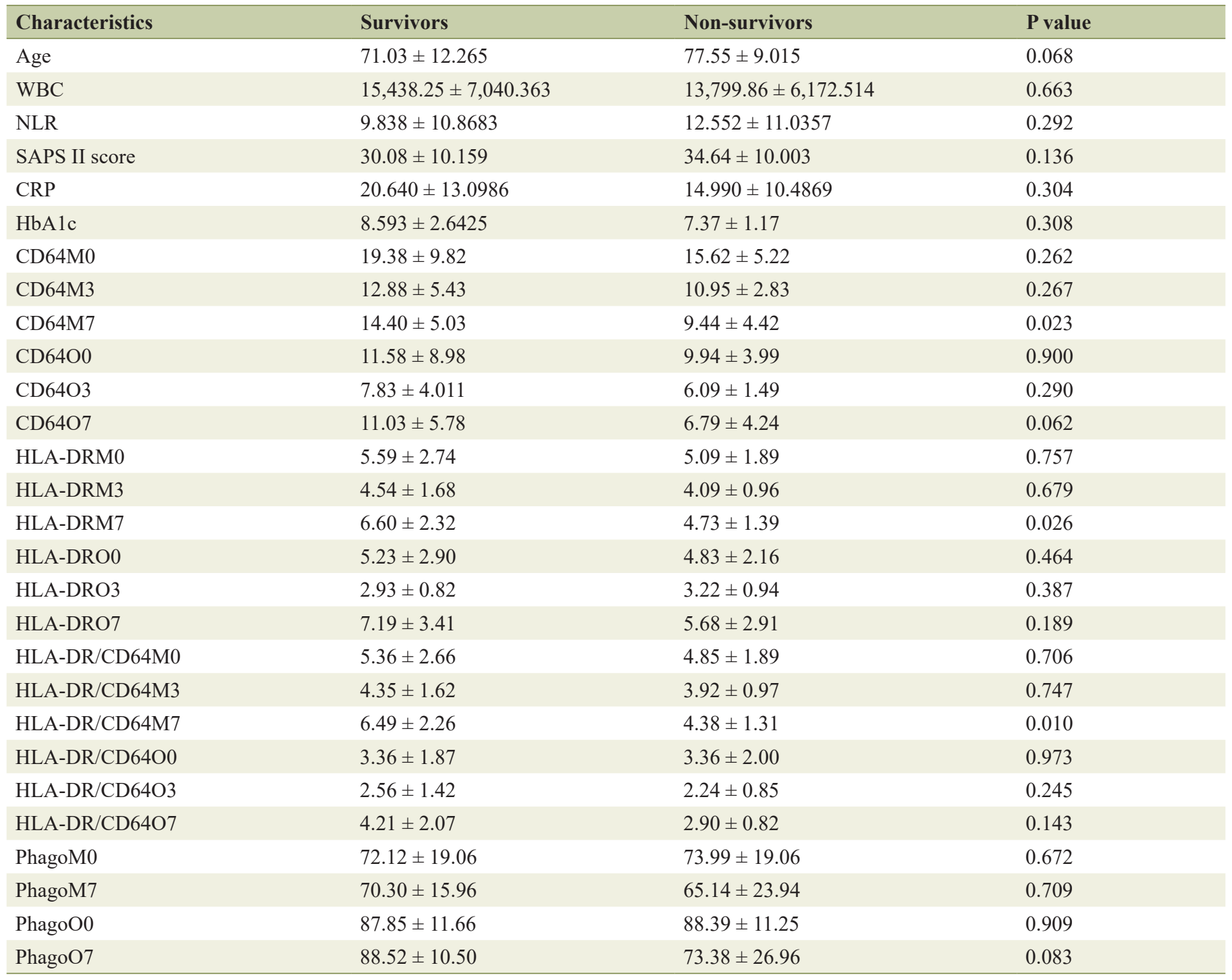

WBC: white blood cells; NLR: neutrophils to lymphocytes ratio; CD64M0: CD64 antigen on monocytes on day 0; CD64M3: CD64 antigen on monocytes on day 3; CD64M7: CD64 antigen on monocytes on day 7; CD64O0: CD64 antigen on neutrophils on day 0; CD64O3: CD64 antigen on neutrophils on day 3; CD64O7: CD64 antigen on neutrophils on day 7; HLA-DRM0: HLA-DR antigen on monocytes on day 0; HLA-DRM3: HLA-DR antigen on monocytes on day 3; HLA-DRM7: HLA-DR antigen on monocytes on day 7; HLA-DRO0: HLA-DR antigen on neutrophils on day 0; HLA-DRO3: HLA-DR antigen on neutrophils on day 3; HLA-DRO7: HLA-DR antigen on neutrophils on day 7; HLA-DR/CD64M0: HLA-DR/CD64 co-expression on monocytes on day 0; HLA-DR/CD64M3: HLA-DR/CD64 co-expression on monocytes on day 3; HLA-DR/CD64M7: HLA-DR/CD64 co-expression on monocytes on day 7; HLA-DR/CD64O0: HLA-DR/CD64 co-expression on neutrophils on day 0; HLA-DR/CD64O3: HLA-DR/CD64 co-expression on neutrophils on day 3; HLA-DR/CD64O7: HLA-DR/CD64 co-expression on neutrophils on day 7; phagoM0: percentage of phagocytosis in monocytes on day 0; phagoM7: percentage of phagocytosis in monocytes on day 7; phagoO0: percentage of phagocytosis in neutrophils on day 0; phagoO7: percentage of phagocytosis in neutrophils on day 7.

markers CD64M, HLA-DRM and HLA-DR/CD64M on day 7 , and that was statistically significant with $P$ values of 0.023 , 0.026 and 0.010 respectively (Fig. 1). This is related to the underlying immune status of the patients as the increased expression of these markers is associated with higher resistance to infections. With regards to literature, this finding is in accordance with a previous study referring to sepsis population [21].

We did not find a significant impact of the type of infection, glycemic control, inflammatory markers on admission or the phagocytosis rate on the survival in the hospitalized diabetics who developed sepsis. However, a borderline but no statistically significant association of Simplified Acute Physiology Score (SAPS) II with survival was documented $(\mathrm{P}=0.057)$.

We found a reduced expression of CD64O $(\mathrm{P}=0.038)$, HLA-DRM $(\mathrm{P}=0.052)$ and the co-expression of CD64/HLA$\mathrm{DR}$ on monocytes on admission $(\mathrm{P}=0.05)$ in diabetics with sepsis and poor glycemic control ( $\mathrm{HbAlc}>8.5)$, compared to diabetics with sepsis and good $(\mathrm{HbA} 1 \mathrm{c}<7)$ or moderate gly- 
Table 3. Analysis of Characteristics in Septic Diabetic Patients With Excellent, Moderate and Inadequate Blood Glucose Control

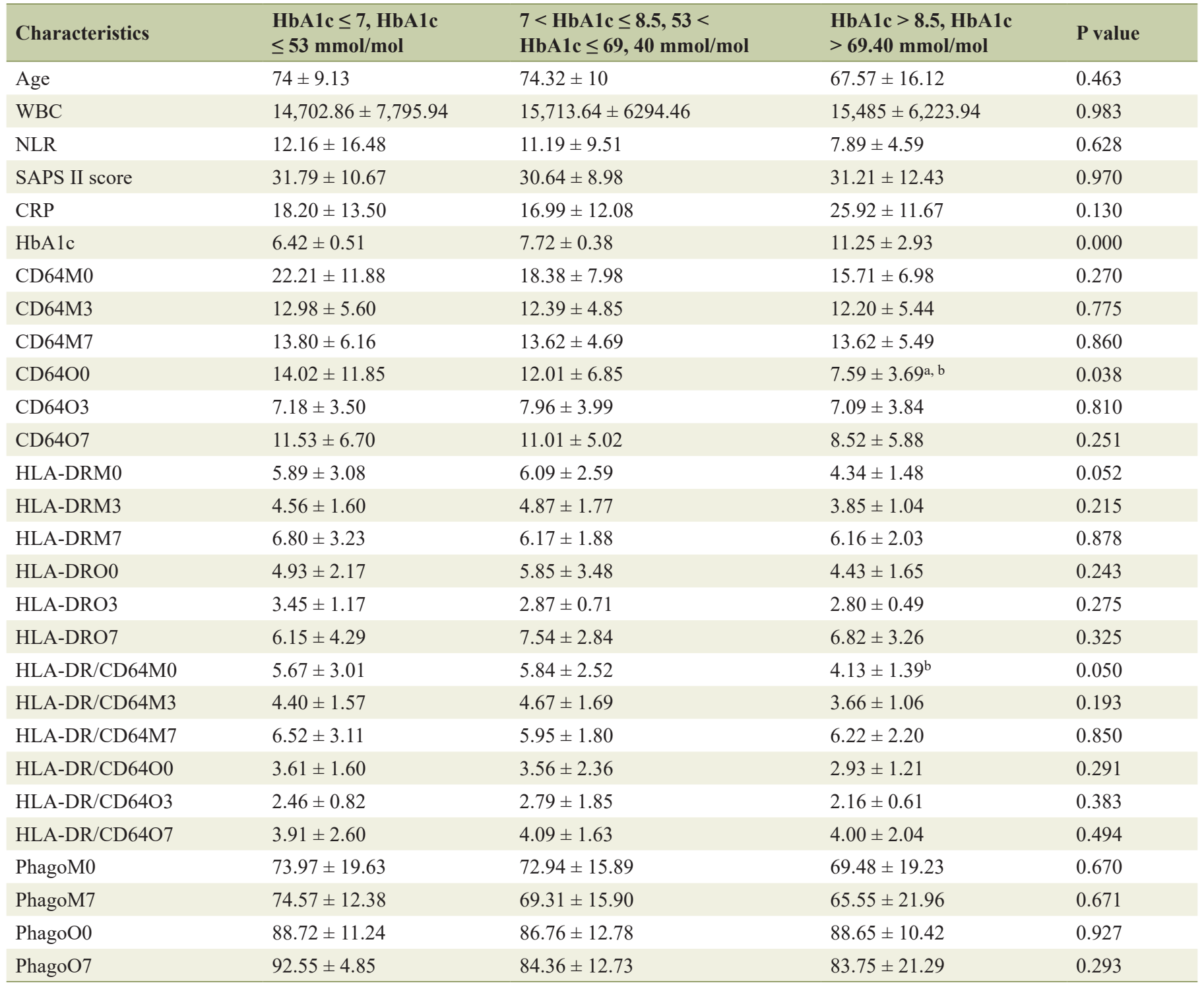

NSGP values: $\mathrm{HbA} 1 \mathrm{c} \leq 7,7<\mathrm{HbA} 1 \mathrm{c} \leq 8.5, \mathrm{HbA} 1 \mathrm{c}>8.5$, respectively; IFCC values in mmol/mol: $\mathrm{HbA} 1 \mathrm{c} \leq 53,53<\mathrm{HbA} 1 \mathrm{c} \leq 69.40, \mathrm{HbA} 1 \mathrm{c}>69.40$, respectively. NSGP: National Glycohemoglobin Standardization Program; IFCC: The International Federation of Clinical Chemistry and Laboratory Medicine; WBC: white blood cells; NLR: neutrophils to lymphocytes ratio; CD64M0: CD64 antigen on monocytes on day 0; CD64M3: CD64 antigen on monocytes on day 3; CD64M7: CD64 antigen on monocytes on day 7; CD64O0: CD64 antigen on neutrophils on day 0; CD64O3: CD64 antigen on neutrophils on day 3; CD6407: CD64 antigen on neutrophils on day7; HLA-DRM0: HLA-DR antigen on monocytes on day 0; HLA-DRM3: HLA-DR antigen on monocytes on day3; HLA-DRM7: HLA-DR antigen on monocytes on day 7; HLA-DRO0: HLA-DR antigen on neutrophils on day 0; HLA-DRO3: HLA-DR antigen on neutrophils on day 3; HLA-DRO7: HLA-DR antigen on neutrophils on day7; HLA-DR/CD64M0: HLA-DR/CD64 co-expression on monocytes on day 0; HLA-DR/CD64M3: HLA-DR/CD64 co-expression on monocytes on day 3; HLA-DR/CD64M7: HLA-DR/CD64 co-expression on monocytes on day 7; HLA-DR/CD64O0: HLA-DR/CD64 co-expression on neutrophils on day 0; HLA-DR/CD64O3: HLA-DR/CD64 co-expression on neutrophils on day3; HLA-DR/CD64O7: HLA-DR/CD64 co-expression on neutrophils on day 7; phagoM0: percentage of phagocytosis in monocytes on day 0; phagoM7: percentage of phagocytosis in monocytes on day 7; phagoO0: percentage of phagocytosis in neutrophils on day 0; phagoO7: percentage of phagocytosis in neutrophils on day 7 . a $<<0.05$ vs. grl. ${ }^{b} P<0.05$ vs. grll.

cemic control $(7<\mathrm{HbA1c}<8.5)$. These findings are consistent with the increased susceptibility of diabetics to infections especially in the cases with poor glycemic control. No association between glycemic control and phagocytosis rates was identified, as in the study by Lin where it was shown that poor glycemic control plays a role in impairing neutrophil phagocy- tosis of capsular serotypes K1/K2 Klebsiella pneumoniae [22].

Although this is a study with a relatively small sample size, some useful information can be extracted with regards to the immunologic reaction of diabetics who suffered from sepsis. In this case series a prognostic relation was found between the studied markers CD64 and HLA-DR of monocytes and sur- 
Table 4. Analysis of Characteristics Between Septic Patients With Diabetes Mellitus and Diabetic Patients Without Sepsis

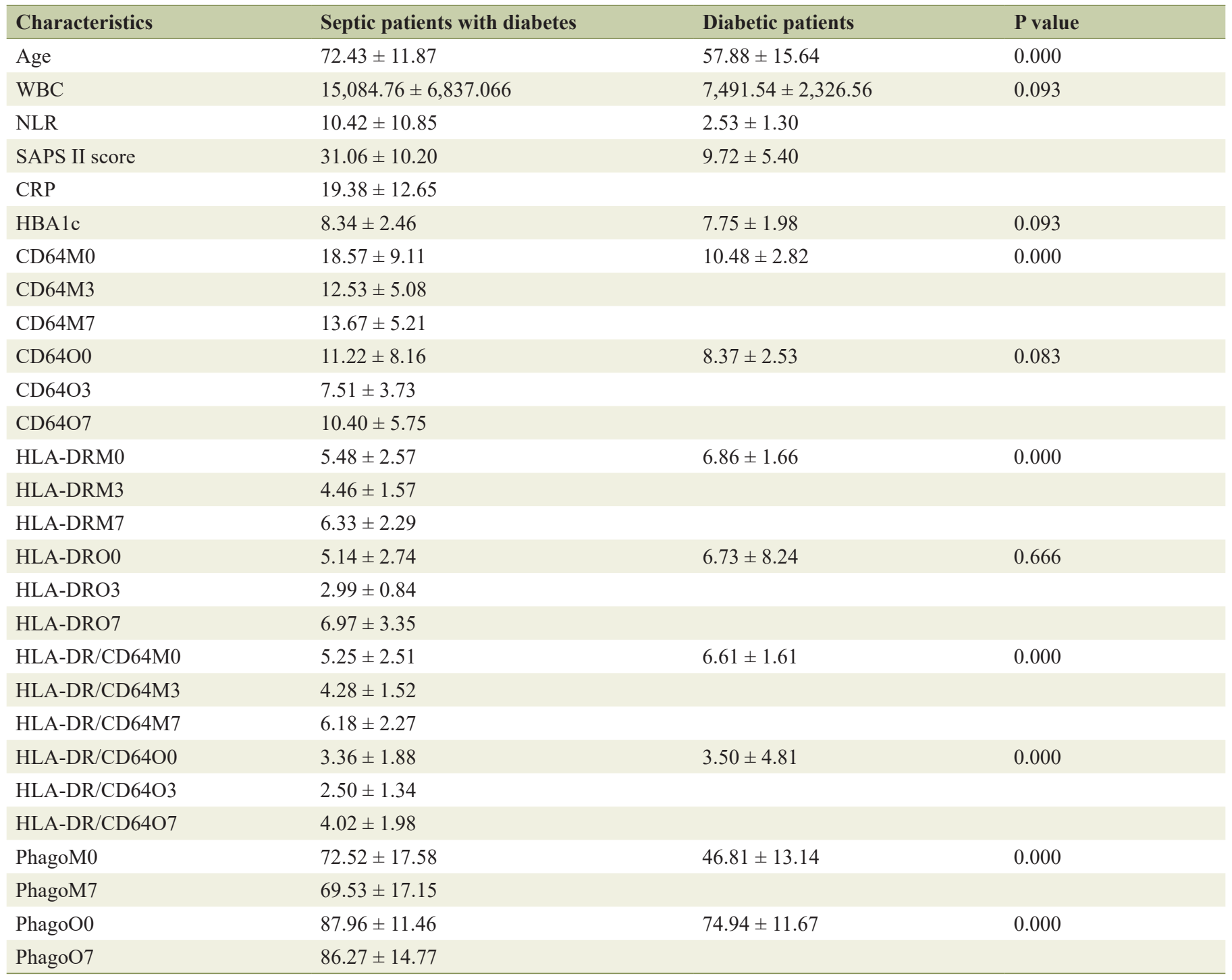

WBC: white blood cells; NLR: neutrophils to lymphocytes ratio; CD64MO: CD64 antigen on monocytes on day 0; CD64M3: CD64 antigen on monocytes on day 3; CD64M7: CD64 antigen on monocytes on day 7; CD64O0: CD64 antigen on neutrophils on day 0; CD64O3: CD64 antigen on neutrophils on day3; CD64O7: CD64 antigen on neutrophils on day7; HLA-DRM0: HLA-DR antigen on monocytes on day 0; HLA-DRM3: HLA-DR antigen on monocytes on day 3; HLA-DRM7: HLADR antigen on monocytes on day7; HLA-DRO0: HLA-DR antigen on neutrophils on day 0; HLA-DRO3: HLA-DR antigen on neutrophils on day 3; HLA-DRO7: HLA-DR antigen on neutrophils on day 7; HLA-DR/CD64M0: HLA-DR/CD64 co-expression on monocytes on day 0; HLA-DR/CD64M3: HLA-DR/CD64 co-expression on monocytes on day 3; HLA-DR/CD64M7: HLA-DR/CD64: co-expression on monocytes on day 7; HLADR/CD64O0: HLA-DR/CD64 co-expression on neutrophils on day 0; HLA-DR/CD64O3: HLA-DR/CD64 co-expression on neutrophils on day 3; HLA-DR/CD64O7: HLA-DR/CD64 co-expression on neutrophils on day 7; phagoM0: percentage of phagocytosis in monocytes on day 0; phagoM7: percentage of phagocytosis in monocytes on day 7; phagoO0: percentage of phagocytosis in neutrophils on day 0; phagoO7: percentage of phagocytosis in neutrophils on day 7.

vival, whereas no relation between type of infection, glycemic control and the outcome was found. Especially, with regards to antigenic expression although this was reduced in both neutrophils and monocytes during the first days of infection, no statistical significance with outcome was noticed. Also, the present study failed to document a relationship between the type of infection with inflammatory markers on admission and the expression of cell surface markers (CD64, HLA-DR, coexpression CD64/HLA-DR, and rate of phagocytosis in neu- trophils (phagoO) and phagocytosis in monocytes (phagoM)). There are many tools in the assessment of the complex underlying pathophysiology in sepsis nowadays including the assessment of the complement pathway and the cytokine profile which could be proved more useful also as prognostic factors. Towards this direction, perhaps the study of other parameters such as complement and cytokine profile could prove to be more useful as prognostic factors. Complement 3 receptor expression has been recorded to be reduced in diabetics with type 

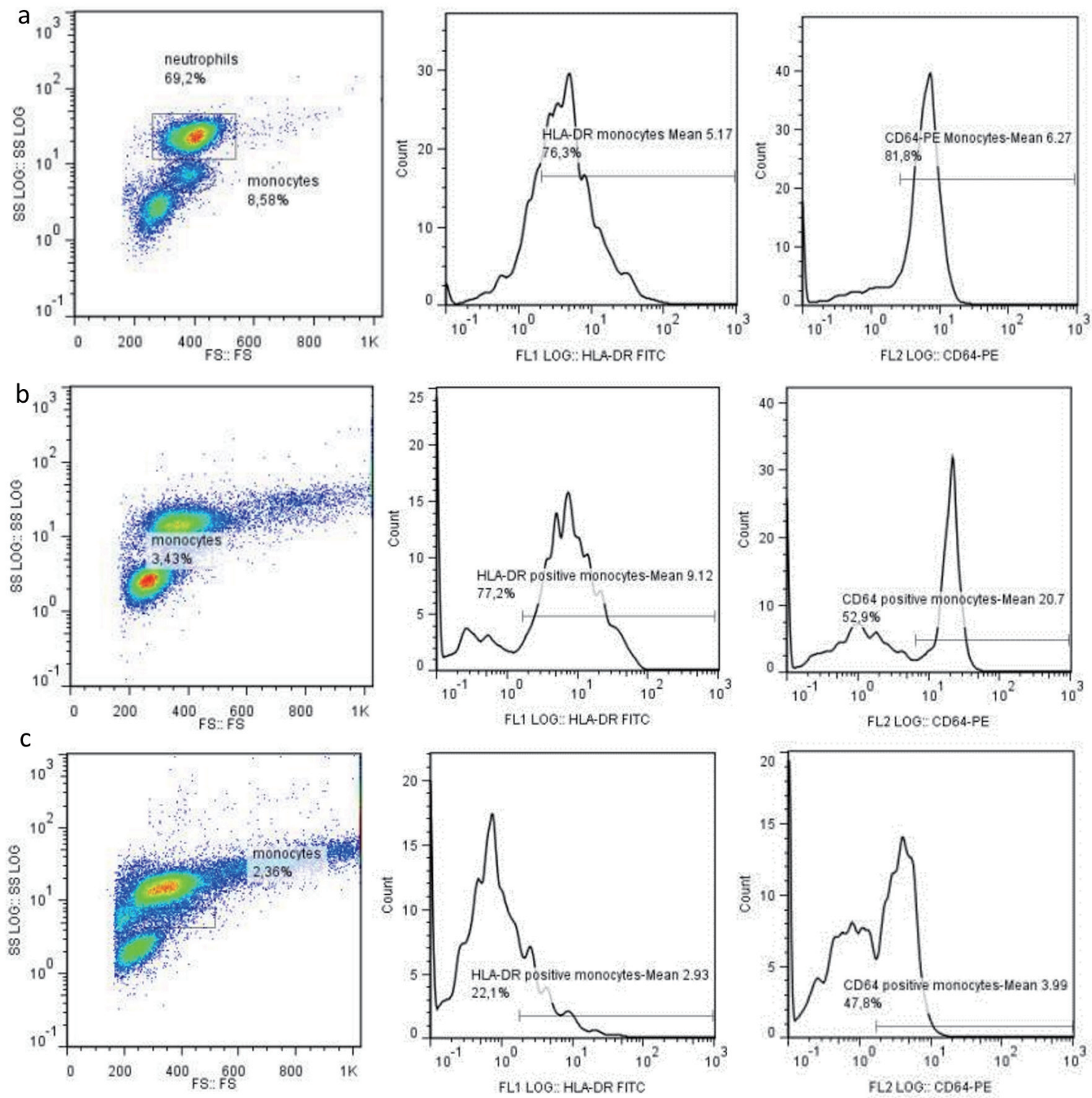

Figure 1. Cytometry flow analysis in a control patient (a), a patient with diabetes and sepsis that survived (b), and a patient that deceased (c), showing the expression of HLA-DR and CD64 on monocytes and neutrophils on day 7.

2 diabetes compared to non-diabetics [23], and also the role of cytokines in T1DM and T2DM is investigated [24].

\section{Conclusions}

The combination of the immune alterations in diabetes with the immune dysregulation during the process of the sepsis syndrome offers a field of interest for tailored interventions. A research effort now is focused on the interplay between sepsis, diabetes and immunity and their impact on survival. This is a small case series indicating alterations of neutrophils and monocytes in diabetics suffering from sepsis at least in the initial phase of acute infection. Large prospective studies are needed to investigate the immune alterations of high risk for population of diabetics who develop sepsis.

\section{Acknowledgments}

None to declare.

\section{Financial Disclosure}

This work was supported entirely by department founds, without any financial support by industry or by any external grants. 


\section{Conflict of Interest}

The authors declare that they do not have any conflict of interest to report.

\section{Informed Consent}

All enrolled patients gave consent for participating in the study.

\section{Author Contributions}

KL collected the samples of the patients, did the laboratory work and wrote the paper; NR did the statistical analysis; MK supervised the laboratory work; MM, CG and DV wrote the paper.

\section{Data Availability}

The authors declare that data supporting the findings of this study are available within the article.

\section{References}

1. Fleischmann C, Scherag A, Adhikari NK, Hartog CS, Tsaganos T, Schlattmann P, Angus DC, et al. Assessment of global incidence and mortality of hospital-treated sepsis. current estimates and limitations. Am J Respir Crit Care Med. 2016;193(3):259-272.

2. Gaieski DF, Edwards JM, Kallan MJ, Carr BG. Benchmarking the incidence and mortality of severe sepsis in the United States. Crit Care Med. 2013;41(5):1167-1174.

3. Frydrych LM, Fattahi F, He K, Ward PA, Delano MJ. Diabetes and sepsis: risk, recurrence, and ruination. Front Endocrinol (Lausanne). 2017;8:271.

4. Cassatella MA. Neutrophil-derived proteins: selling cytokines by the pound. Adv Immunol. 1999;73:369-509.

5. Hatanaka E, Monteagudo PT, Marrocos MS, Campa A. Neutrophils and monocytes as potentially important sources of proinflammatory cytokines in diabetes. Clin Exp Immunol. 2006;146(3):443-447.

6. Baggiolini M, Dewald B, Moser B. Human chemokines: an update. Annu Rev Immunol. 1997;15:675-705.

7. Singer M, Deutschman CS, Seymour CW, Shankar-Hari M, Annane D, Bauer M, Bellomo R, et al. The third international consensus definitions for sepsis and septic shock (Sepsis-3). JAMA. 2016;315(8):801-810.

8. Brownlee M. Biochemistry and molecular cell biology of diabetic complications. Nature. 2001;414(6865):813820 .

9. Hameed I, Masoodi SR, Mir SA, Nabi M, Ghazanfar K, Ganai BA. Type 2 diabetes mellitus: From a metabolic disorder to an inflammatory condition. World J Diabetes. 2015;6(4):598-612.

10. Geerlings SE, Brouwer EC, Van Kessel KC, Gaastra W,
Stolk RP, Hoepelman AI. Cytokine secretion is impaired in women with diabetes mellitus. Eur J Clin Invest. 2000;30(11):995-1001.

11. Peleg AY, Weerarathna T, McCarthy JS, Davis TM. Common infections in diabetes: pathogenesis, management and relationship to glycaemic control. Diabetes Metab Res Rev. 2007;23(1):3-13.

12. Schubert S, Heesemann J. [Infections in diabetes mellitus]. Immun Infekt. 1995;23(6):200-204.

13. Pozzilli P, Leslie RD. Infections and diabetes: mechanisms and prospects for prevention. Diabet Med. 1994;11(10):935-941.

14. Itariu BK, Stulnig TM. Autoimmune aspects of type 2 diabetes mellitus - a mini-review. Gerontology. 2014;60(3):189-196.

15. Alexandraki KI, Piperi C, Ziakas PD, Apostolopoulos NV, Makrilakis K, Syriou V, Diamanti-Kandarakis E, et al. Cytokine secretion in long-standing diabetes mellitus type 1 and 2: associations with low-grade systemic inflammation. J Clin Immunol. 2008;28(4):314-321.

16. Li P, Li M, Lindberg MR, Kennett MJ, Xiong N, Wang Y. PAD4 is essential for antibacterial innate immunity mediated by neutrophil extracellular traps. J Exp Med. 2010;207(9):1853-1862.

17. Brinkmann V, Reichard U, Goosmann C, Fauler B, Uhlemann Y, Weiss DS, Weinrauch Y, et al. Neutrophil extracellular traps kill bacteria. Science. 2004;303(5663):15321535.

18. Wong SL, Demers M, Martinod K, Gallant M, Wang Y, Goldfine AB, Kahn CR, et al. Diabetes primes neutrophils to undergo NETosis, which impairs wound healing. Nat Med. 2015;21(7):815-819.

19. Docke WD, Randow F, Syrbe U, Krausch D, Asadullah $\mathrm{K}$, Reinke P, Volk HD, et al. Monocyte deactivation in septic patients: restoration by IFN-gamma treatment. Nat Med. 1997;3(6):678-681.

20. Carson WF, Cavassani KA, Dou Y, Kunkel SL. Epigenetic regulation of immune cell functions during post-septic immunosuppression. Epigenetics. 2011;6(3):273-283.

21. Danikas DD, Karakantza M, Theodorou GL, Sakellaropoulos GC, Gogos CA. Prognostic value of phagocytic activity of neutrophils and monocytes in sepsis. Correlation to CD64 and CD14 antigen expression. Clin Exp Immunol. 2008;154(1):87-97.

22. Lin JC, Siu LK, Fung CP, Tsou HH, Wang JJ, Chen CT, Wang SC, et al. Impaired phagocytosis of capsular serotypes K1 or K2 Klebsiella pneumoniae in type 2 diabetes mellitus patients with poor glycemic control. J Clin Endocrinol Metab. 2006;91(8):3084-3087.

23. Ly J, Morris D, Lagman M, Ng C, Anderson S, Daliva $\mathrm{J}$, Muwanas N, et al. Complement 3 receptor expression in individuals with type 2 diabetes. Recent Pat Antiinfect Drug Discov. 2016;11(2):174-182.

24. Chatzigeorgiou A, Harokopos V, Mylona-Karagianni C, Tsouvalas E, Aidinis V, Kamper EF. The pattern of inflammatory/anti-inflammatory cytokines and chemokines in type 1 diabetic patients over time. Ann Med. 2010;42(6):426-438. 